\title{
Comparison of Ticlopidine and Cilostazol for the Prevention of Restenosis After Percutaneous Transluminal Coronary Angioplasty
}

\author{
Noriyasu NagaokA, MD, Tatsuaki Matsubara, MD, Katsuo OKAZAKI, ${ }^{1}$ MD, \\ Naomichi MASUdA, ${ }^{1}$ MD, Kyoko SHIKAURA, ${ }^{1} \mathrm{MD}$, and Nigishi HOTTA, MD
}

\begin{abstract}
SUMMARY
Prevention of restenosis after percutaneous transluminal coronary angioplasty (PTCA) continues to be a significant problem. Recent controlled studies have demonstrated that cilostazol suppresses restenosis after PTCA. The effects of ticlopidine, another antiplatelet agent, were compared in terms of outcomes of patients randomized for treatment with the two drugs after PTCA. A total of 35 patients (47 lesions) were assigned prospectively and randomly to ticlopidine (17 patients, 24 lesions) and cilostazol (18 patients, 23 lesions) groups. Minimal luminal diameter (MLD) and percentage of stenosis to reference diameter were estimated before PTCA, just after the procedure and after 4 months follow-up. All patients underwent 4 months angiographic follow-up, at the end of which MLD was $2.03 \pm 0.71 \mathrm{~mm}$ in the ticlopidine group and $2.05 \pm 0.68 \mathrm{~mm}$ in the cilostazol group $(p=0.95)$, and the percentage of stenosis to reference diameter was $31.4 \pm$ $16.7 \%$ and $30.0 \pm 17.0 \%$, respectively $(p=0.78)$. The restenosis rate was $12.5 \%$ in the ticlopidine group and $17.4 \%$ in the cilostazol group $(p=0.69)$, relatively low as compared to the $20 \%$ to $30 \%$ reported in previous studies. Adverse drug reactions during the followup period were observed in two of the ticlopidine group and none of the cilostazol group. We conclude that both ticlopidine and cilostazol are effective for the prevention of restenosis after PTCA, however the former may be associated with slight side effects. (Jpn Heart J 2001; 42: 43-54)
\end{abstract}

Key words: Antiplatelet therapy, Follow-up studies, Quantitative coronary arteriography

RESTENOSIS after percutaneous transluminal coronary angioplasty (PTCA) is still a major limitation to long-term successful therapy. Coronary stent implantation is associated with a significant reduction in the angiographic restenosis rate compared with conventional simple balloon angioplasty, with decreases to $20 \%$ to $30 \%$ reported by the BENESTENT ${ }^{1)}$ and STRESS ${ }^{2)}$ trials, but the search for

From Third Department of Internal Medicine, Nagoya University School of Medicine, Aichi, ${ }^{1}$ Fukuroi Municipal Hospital, Shizuoka, Japan.

Address for correspondence: Tatsuaki Matsubara, MD, Third Department of Internal Medicine, Nagoya University School of Medicine, 65 Tsurumai-cho, Showa-ku, Nagoya, Aichi 466-8550, Japan.

Received for publication July 25, 2000.

Revised and accepted September 29, 2000. 
pharmacological agents capable of causing further reduction continues. Antiplatelet therapy is one leading candidate. Though the mechanisms of restenosis after PTCA have not been thoroughly elucidated, previous studies have suggested that platelet aggregation at the lesion site is a main cause, initiating a chain of events leading to smooth muscle cell proliferation and neointimal hyperplasia. ${ }^{3-5}$ ) Antiplatelet agents hinder platelets from aggregating and one example, cilostazol, has already been shown to prevent restenosis after PTCA in controlled studies. ${ }^{6-9)}$ Ticlopidine is another promising antiplatelet agent, which has similar pharmacological properties. A prospective and randomized comparison of ticlopidine and cilostazol for this purpose was therefore conducted in the present study.

\section{METHODS}

Patients: This study was a prospective and randomized comparison of two antiplatelet drugs: ticlopidine and cilostazol for the prevention of restenosis after PTCA, administered at a daily dose of $200 \mathrm{mg}$ for 4 months from just after the intervention. Both groups of patients were also administrated an $81 \mathrm{mg}$ dose of aspirin daily. Between November 1997 and March 1998, 47 patients (61 lesions) underwent PTCA including stent implantation successfully at Fukuroi Municipal Hospital. Of these, 35 eligible patients (47 lesions) were assigned prospectively and randomly to two groups: 17 patients (24 lesions) for the ticlopidine group, including 12 patients (15 lesions) undergoing stent implantation, and 18 patients (23 lesions) for the cilostazol group, with 9 patients (10 lesions) receiving stents. All patients gave written informed consent as approved by the hospital committee. Of the others, 9 patients (11 lesions) were excluded from the study because they had already been administered ticlopidine, cilostazol, or warfarin before PTCA, and 3 patients ( 3 lesions) were contraindicated for antiplatelet therapy because of a history of gastric ulcer or intracranial hemorrhage.

Study design: All the randomized patients were well without vessel occlusion or any other cardiac events (myocardial infarction, coronary artery bypass graft surgery, or repeat PTCA) during the 4 months follow-up study. Minimal luminal diameter (MLD) and percentage stenosis in comparison to the reference diameter were estimated by the quantitative coronary arteriography (QCA) system on three angiograms: one before the intervention, one after the procedure, and one at the end of the follow-up period.

PTCA was performed using a standard conventional technique after administration of $10,000 \mathrm{U}$ of heparin. When plain balloon angioplasty 
ended in a suboptimal result, stent implantation was carried out. Indications for this were residual stenosis of $\geq 30 \%$ after balloon angioplasty or coronary artery dissections. As a general rule, stent implantation was performed for lesions whose reference diameters were larger than $2.75 \mathrm{~mm}$ without showing evidence of severe calcification on angiograms or excessive angles proximal to the lesion likely to increase the risk of stent dislodgment. Additional noncompliant balloons were used for high-pressure final stent dilatation in the patients with stent implantation. Multiple stents were employed when necessary to cover the full extent of the target lesion or of any dissection. In most patients, the judgement was based solely on visual assessment of the site in angiograms. In some cases, intravascular ultrasound was used for estimating vessel diameters or detecting coronary artery dissections, but this was not part of the standard protocol.

Angiographic analysis: Angiographic analysis was performed by operators who were unaware of which therapy had been chosen. The QCA system used in the present study was centered on a Cardio 500 (Kontron Elektronik, Michael Müller, Munich, Germany). Angiographic images were projected onto a cine $35-\mathrm{mm}$ viewer optically and entered into the system through the video camera. Edge detection of the coronary artery in the target lesion including the proximal and distal normal segments was automatically achieved by the system using image zooming after the centerline was determined. ${ }^{10)}$ Absolute measurements of MLD and the reference diameter were determined using the guide catheter as a scaling device. Percentage stenosis was calculated from the MLD and the reference diameter.

Acute lumen gain was calculated as the difference between MLD before and just after the intervention, and late lumen loss by subtracting the MLD after 4 months follow-up from that just after the intervention. Loss indices were calculated as the ratios of late lumen loss to acute lumen gain. Restenosis was defined as a stenosis $>50 \%$ of the diameter at follow-up angiography.

Statistical analysis: Comparison of discrete variables, expressed as counts, was performed using Fisher's exact test. Continuous variables are expressed as mean \pm SD values and compared with the use of unpaired, two-tailed $t$ tests after a normal distribution was assured. Statistical significance was assumed for $p<0.05$.

\section{RESULTS}

Patients and lesion characteristics: Four months follow-up angiography was 
performed for all eligible patients in both groups. Table I presents the baseline clinical characteristics of the randomized patients who completed the angiographic protocol of this study. All clinical features were evenly distributed between the two groups. Follow-up angiography was performed at comparable time intervals with a mean of $128.4 \pm 15.9$ days for ticlopidine and $124.7 \pm 16.3$ days for cilostazol treated cases $(p=0.50)$.

Table II presents target lesion characteristics. The two groups did not differ significantly with regard to parameters such as target vessel, lesion severity, and number of lesions treated. Technical details of PTCA, such as maximal balloon pressure $(14.0 \pm 3.2$ atoms in the ticlopidine group versus $12.5 \pm 2.6$ atoms in the cilostazol group: $p=0.80)$, total pressure

Table I. Baseline Clinical Characteristics

\begin{tabular}{lccc}
\hline & Ticlopidine group & Cilostazol group & $p$ \\
\hline Age, y & $(n=17)$ & $(n=18)$ & \\
Male & $63.4 \pm 9.6$ & $61.8 \pm 8.9$ & 0.62 \\
Smoking & $12(70.6 \%)$ & $11(61.1 \%)$ & 0.55 \\
Hypercholesterolemia & $10(58.8 \%)$ & $8(44.4 \%)$ & 0.39 \\
Diabetes mellitus & $6(35.3 \%)$ & $5(27.8 \%)$ & 0.55 \\
Hypertension & $3(17.6 \%)$ & $4(22.2 \%)$ & 0.74 \\
Multivessel disease & $10(58.8 \%)$ & $8(44.4 \%)$ & 0.93 \\
Acute myocardial infarction & $5(29.4 \%)$ & $4(22.2 \%)$ & 0.63 \\
Old myocardial infarction & $6(35.3 \%)$ & $5(27.8 \%)$ & 0.63 \\
Unstable angina pectoris & $2(11.8 \%)$ & $1(5.6 \%)$ & 0.51 \\
Stent placement & $2(11.8 \%)$ & $3(16.7 \%)$ & 0.68 \\
Follow-up days & $12(70.6 \%)$ & $9(50.0 \%)$ & 0.21 \\
\hline
\end{tabular}

Age and follow-up days are mean $\pm \mathrm{SD}$; other variables are number. of patients (\%).

Table II. Target Lesion Characteristics

\begin{tabular}{lccc}
\hline & Ticlopidine group & Cilostazol group & $p$ \\
\hline Target lesions & $(n=24)$ & $(n=23)$ & \\
LAD & 11 & 8 & 0.44 \\
LCx & 8 & 7 & 0.83 \\
RCA & 5 & 8 & 0.28 \\
ACC/AHA lesion classification & & & \\
Type A & 12 & 7 & 0.17 \\
Type B & 11 & 5 & 0.89 \\
Type C & 3 & 5 & 0.40 \\
\hline
\end{tabular}

Values are counts. $\mathrm{LAD}=$ left anterior descending coronary artery; $\mathrm{LCx}=$ left circumflex artery; $\mathrm{RCA}=$ right coronary artery; $\mathrm{ACC} / \mathrm{AHA}=$ American College of Cardiology/American Heart Association. 
time $(150.0 \pm 72.2$ seconds versus $134.0 \pm 45.0$ seconds: $p=0.54)$, and stent implantation (15 of 24 PTCA lesions versus 10 of 23 PTCA lesions: $p=0.25)$ were not significantly different.

Data from QCA analysis: Data from QCA analysis are summarized in Table III. Division was made on the basis of whether stent implantation was performed. There were no significant differences in the reference diameter, MLD, and percentage diameter stenosis between the ticlopidine group and the cilostazol group before the intervention, just after the procedure, and at follow-up in the patients with or without stent implantation,

Table III. Data from Quantitative Coronary Arteriography

\begin{tabular}{|c|c|c|c|}
\hline & Ticlopidine group & Cilostazol group & $p$ \\
\hline All lesions & $(n=24)$ & $(n=23)$ & \\
\hline \multicolumn{4}{|l|}{ Before PTCA } \\
\hline Reference diameter, mm & $2.71 \pm 0.55$ & $2.79 \pm 0.52$ & 0.62 \\
\hline MLD, mm & $0.49 \pm 0.41$ & $0.54 \pm 0.35$ & 0.66 \\
\hline Diameter stenosis, $\%$ & $80.6 \pm 14.7$ & $79.9 \pm 12.3$ & 0.87 \\
\hline \multicolumn{4}{|l|}{ Immediately after procedure } \\
\hline Reference diameter, $\mathrm{mm}$ & $2.87 \pm 0.53$ & $2.92 \pm 0.52$ & 0.78 \\
\hline MLD, $\mathrm{mm}$ & $2.46 \pm 0.55$ & $2.39 \pm 0.52$ & 0.64 \\
\hline Diameter stenosis, $\%$ & $14.6 \pm 6.53$ & $18.2 \pm 9.40$ & 0.13 \\
\hline \multicolumn{4}{|l|}{ After 4-months follow-up } \\
\hline Reference diameter, mm & $2.79 \pm 0.57$ & $2.79 \pm 0.54$ & 0.99 \\
\hline MLD, $\mathrm{mm}$ & $2.03 \pm 0.71$ & $2.05 \pm 0.68$ & 0.95 \\
\hline Diameter stenosis, $\%$ & $31.4 \pm 16.7$ & $30.0 \pm 17.0$ & 0.78 \\
\hline Lesions with stent implantation & $(n=15)$ & $(n=10)$ & \\
\hline \multicolumn{4}{|l|}{ Before PTCA } \\
\hline Reference diameter, $\mathrm{mm}$ & $2.86 \pm 0.54$ & $3.00 \pm 0.52$ & 0.53 \\
\hline MLD, mm & $0.48 \pm 0.47$ & $0.61 \pm 0.40$ & 0.49 \\
\hline Diameter stenosis, $\%$ & $82.1 \pm 16.1$ & $79.6 \pm 11.4$ & 0.68 \\
\hline \multicolumn{4}{|l|}{ Immediately after procedure } \\
\hline Reference diameter, mm & $3.02 \pm 0.50$ & $3.22 \pm 0.44$ & 0.32 \\
\hline MLD, mm & $2.59 \pm 0.53$ & $2.64 \pm 0.50$ & 0.83 \\
\hline Diameter stenosis, \% & $14.7 \pm 5.96$ & $18.3 \pm 9.33$ & 0.28 \\
\hline \multicolumn{4}{|l|}{ After 4-months follow-up } \\
\hline Reference diameter, $\mathrm{mm}$ & $2.93 \pm 0.56$ & $3.03 \pm 0.52$ & 0.66 \\
\hline MLD, mm & $2.10 \pm 0.80$ & $2.35 \pm 0.74$ & 0.44 \\
\hline Diameter stenosis, $\%$ & $33.6 \pm 19.9$ & $32.3 \pm 19.1$ & 0.38 \\
\hline Lesions without stent implantation & $(n=9)$ & $(n=13)$ & \\
\hline \multicolumn{4}{|l|}{ Before PTCA } \\
\hline Reference diameter, $\mathrm{mm}$ & $2.47 \pm 0.52$ & $2.63 \pm 0.48$ & 0.45 \\
\hline MLD, mm & $0.51 \pm 0.30$ & $0.49 \pm 0.31$ & 0.87 \\
\hline Diameter stenosis, $\%$ & $78.1 \pm 12.5$ & $80.1 \pm 13.5$ & 0.72 \\
\hline \multicolumn{4}{|l|}{ Immediately after procedure } \\
\hline Reference diameter, $\mathrm{mm}$ & $2.62 \pm 0.51$ & $2.68 \pm 0.47$ & 0.78 \\
\hline MLD, mm & $2.25 \pm 0.50$ & $2.20 \pm 0.47$ & 0.81 \\
\hline Diameter stenosis, \% & $14.3 \pm 7.76$ & $18.1 \pm 9.34$ & 0.33 \\
\hline \multicolumn{4}{|l|}{ After 4-months follow-up } \\
\hline Reference diameter, $\mathrm{mm}$ & $2.54 \pm 0.42$ & $2.60 \pm 5.00$ & 0.79 \\
\hline MLD, mm & $1.93 \pm 0.55$ & $1.82 \pm 0.54$ & 0.64 \\
\hline Diameter stenosis, $\%$ & $27.7 \pm 8.77$ & $32.3 \pm 19.1$ & 0.51 \\
\hline
\end{tabular}

Values are mean \pm SD or percentages of the target lesions. PTCA $=$ percutaneous transluminal coronary angioplasty; MLD = minimal luminal diameter. 
or for total cases. Figure 1 displays changes of MLD for total patients in both groups. Figure 2 illustrates acute lumen gain as well as late lumen loss. There were no significant differences in acute lumen gain and late lumen loss irrespective of stent implantation, as shown in Table IV.

Restenosis rate: Restenosis was observed with 3 lesions in the ticlopidine group $(12.5 \%)$ and 4 lesions in the cilostazol group $(17.4 \%, p=0.69)$, all three and one, respectively, with stent implantation. The $p$ values between the two groups with and without stent implantation were $p=0.51(12.5 \%$ in the ticlopidine group versus $10.0 \%$ in the cilostazol), and $p=0.24(0 \%$ versus $23.1 \%$ ).

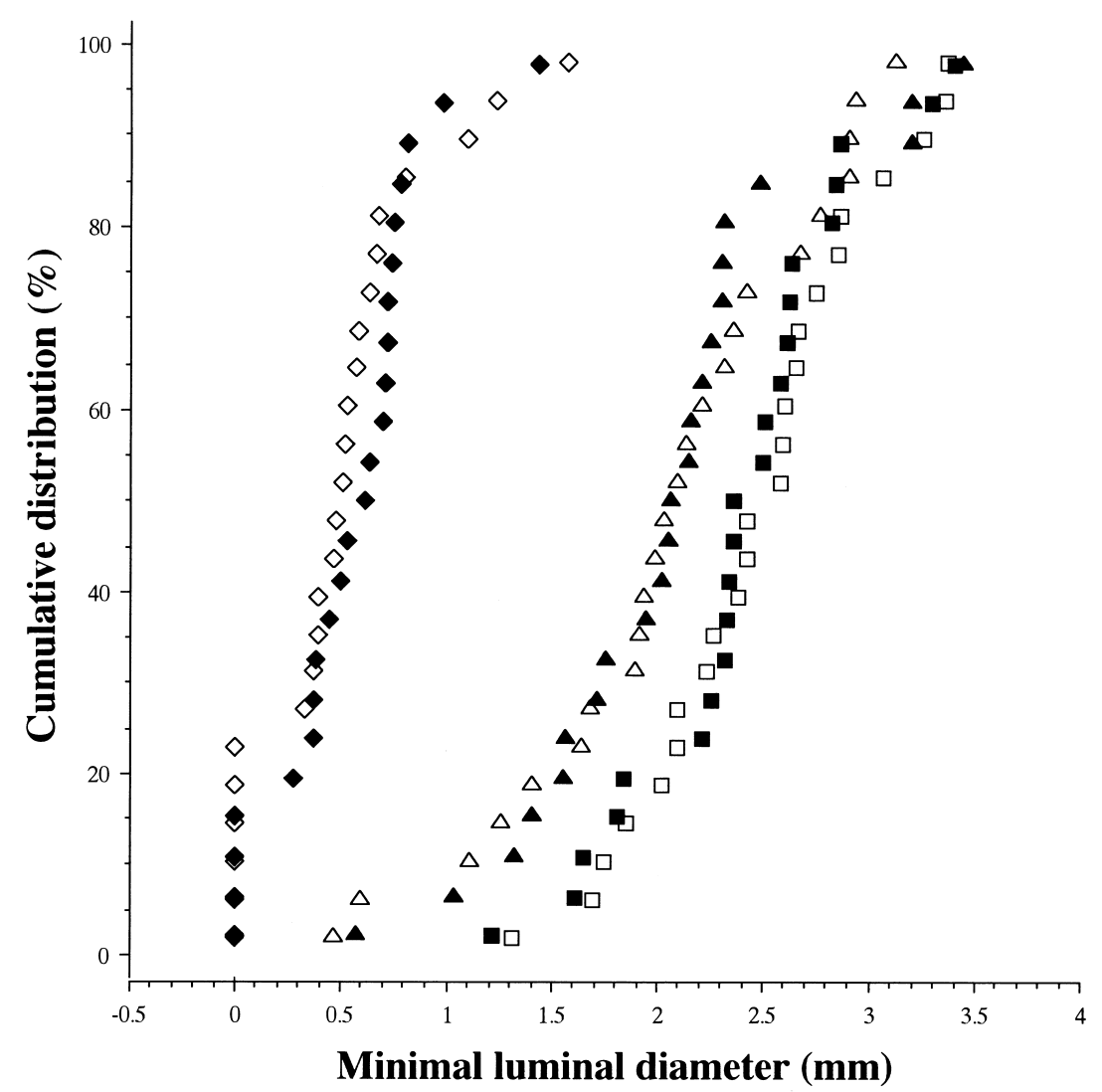

Figure 1. Cumulative distribution curves for minimal luminal diameter (MLD) before (diamonds), just after intervention (squares) and after 4-month angiographic follow-up (triangles) in both treatment groups. Open and closed symbols indicate ticlopidine and cilostazol groups, respectively. There were no significant differences in MLD before, just after intervention or after 4-month follow-up between the two groups. 


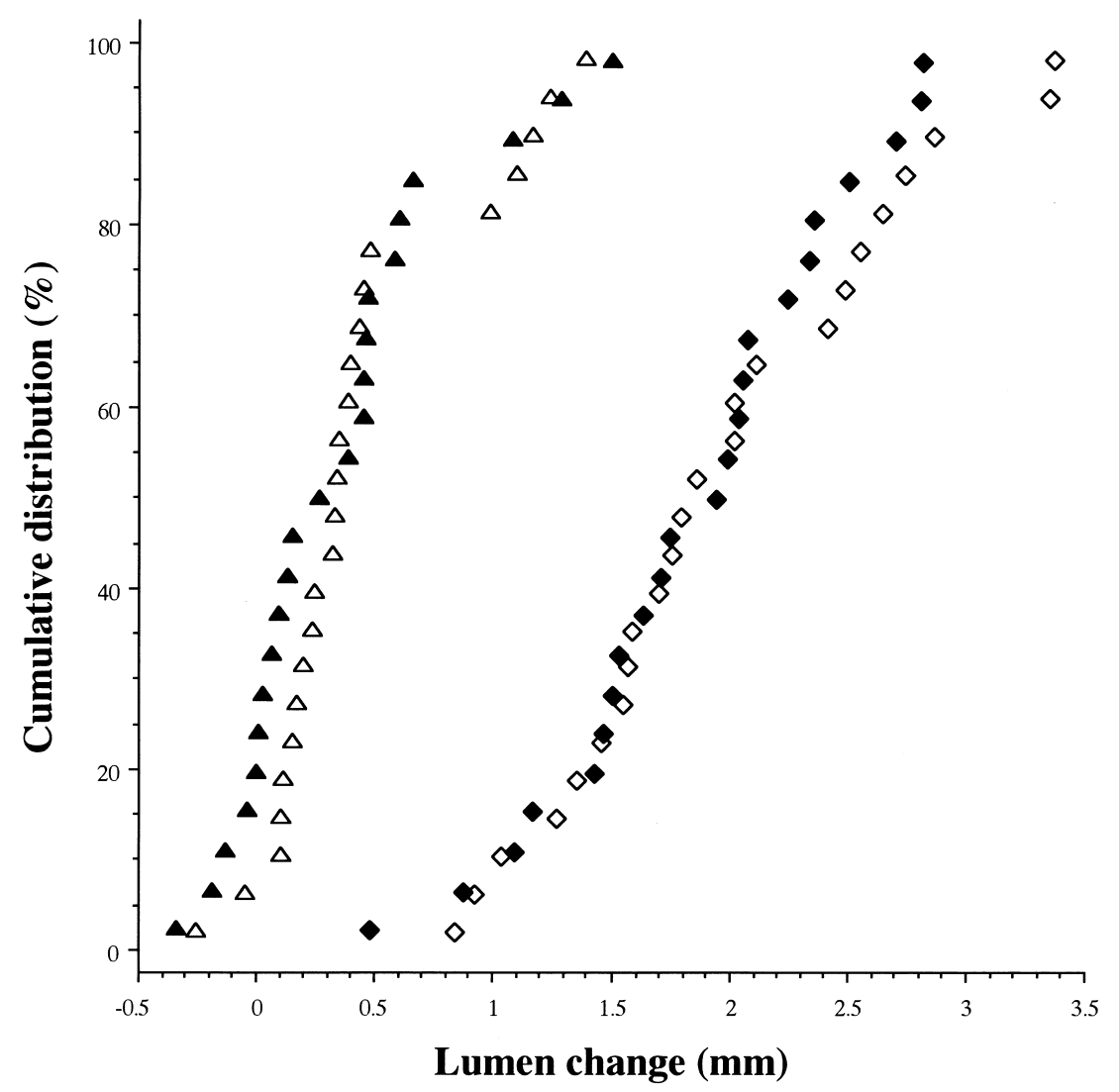

Figure 2. Cumulative distribution curves for acute lumen gain just after intervention (diamonds) and late lumen loss after 4-month angiographic follow-up (triangles) in both treatment groups. Open and closed symbols indicate ticlopidine and cilostazol groups, respectively. There were no significant differences in acute lumen gain or late lumen loss between the two groups.

Table IV. Lumen Changes

\begin{tabular}{lccc}
\hline & Ticlopidine group & Cilostazol group & $p$ \\
\hline All lesions & $(n=24)$ & $(n=23)$ & \\
Acute lumen gain, mm & $1.97 \pm 0.71$ & $1.85 \pm 0.62$ & 0.53 \\
Late lumen loss, mm & $0.43 \pm 0.43$ & $0.35 \pm 0.46$ & 0.51 \\
Loss index & $0.26 \pm 0.30$ & $0.19 \pm 0.33$ & 0.48 \\
Lesions with stent implantation & $(n=15)$ & $(n=10)$ & \\
Acute lumen gain, mm & $2.11 \pm 0.77$ & $2.03 \pm 0.55$ & 0.77 \\
Late lumen loss, mm & $0.49 \pm 0.49$ & $0.29 \pm 0.30$ & 0.26 \\
Loss index & $0.29 \pm 0.35$ & $0.16 \pm 0.20$ & 0.31 \\
Lesions without stent implantation & $(n=9)$ & $(n=13)$ & \\
Acute lumen gain, mm & $1.74 \pm 0.57$ & $1.71 \pm 0.65$ & 0.91 \\
Late lumen loss, mm & $0.33 \pm 0.29$ & $0.39 \pm 0.57$ & 0.78 \\
Loss index & $0.21 \pm 0.19$ & $0.22 \pm 0.41$ & 0.96
\end{tabular}

Values are mean \pm SD. Loss index means the ratio of the late lumen loss to the acute lumen gain. 
Table V. Cardiac and Noncardiac Events

\begin{tabular}{lcc}
\hline & Ticlopidine group & Cilostazol group \\
\hline Cardiac events & $(n=17)$ & $(n=18)$ \\
Noncardiac events & $0(0 \%)$ & $0(0 \%)$ \\
Intracranial hemorrhage & $0(0 \%)$ & $0(0 \%)$ \\
Bleeding requiring transfusion & $0(0 \%)$ & $0(0 \%)$ \\
Leukopenia & $0(0 \%)$ & $0(0 \%)$ \\
Thrombocytopenia & $0(0 \%)$ & $0(0 \%)$ \\
Elevated aminotransferase & $1(5.9 \%)$ & $0(0 \%)$ \\
Skin rash & $1(5.9 \%)$ & $0(0 \%)$ \\
Gastrointestinal disturbance & $0(0 \%)$ & $0(0 \%)$ \\
\hline
\end{tabular}

Values are counts (\%). There were no significant differences between the two groups. Cardiac events are vessel occlusion, myocardial infarction, coronary bypass surgery and repeat PTCA.

Safety: All events during the follow-up period are summarized in Table V. Cardiac events, including vessel occlusion, myocardial infarction, coronary bypass surgery and repeat PTCA did not occur. Noncardiac events were observed in two cases in the ticlopidine group and in none in the cilostazol group $(p=0.23)$. One patient demonstrated elevated aminotransferase between $50 \mathrm{IU} / l$ and $100 \mathrm{IU} / l$, which later normalized without treatment. The other had transient tolerable skin rashes on the legs. In neither case did this make continued administration difficult.

\section{DISCUSSION}

The present prospective and randomized trial of restenosis indices at 4 months, comparing two different antiplatelet drugs, ticlopidine versus cilostazol, revealed restenosis rates of $12.5 \%$ and $17.4 \%$, respectively. The values are appreciably lower than the $22 \%$ and $31.6 \%$ reported after stent implantation in the BENESTENT ${ }^{1)}$ and STRESS ${ }^{2}$ studies. In earlier controlled studies of cilostazol with placebo groups, restenosis rates of $17 \%$ and $17.9 \%$ after balloon angioplasty were reported with cilostazol as compared to $40 \%$ and $39.5 \%$ in the controls. ${ }^{6,9)}$ The rates in the present study are thus in line with the literature in demonstrating an advantage with antiplatelet drugs, further suggesting that both ticlopidine and cilostazol are effective for reducing restenosis after PTCA.

Restenosis generally occurs in more than one third of patients after PTCA, ${ }^{11)}$ presenting as a significant cause of morbidity with high medical 
costs related to repeat intervention. In response to wall stretch and injury by balloon dilatation, elastic recoil, ${ }^{12}$ neointimal hyperplasia, ${ }^{3-5)}$ and arterial remodeling ${ }^{13)}$ contribute to coronary luminal renarrowing during the first few months after PTCA. Various approaches to reduce restenosis have been tested. The aim of pharmacological treatment has been the inhibition of mechanisms responsible for the fibrointimal hyperplasia leading to neointimal formation at PTCA sites. Though the underlying processes have not yet been fully elucidated, a special role in promoting the migration and proliferation of smooth muscle cells has been advocated for adherent platelets and resulting mural thrombi. ${ }^{3-5)}$ While previous studies with antiplatelet and anticoagulant agents such as aspirin, ${ }^{14)}$ aspirin plus dipyridamole, ${ }^{15}$ ) warfarin, ${ }^{16)}$ heparin, ${ }^{17)}$ thromboxane $\mathrm{A}_{2}$ receptor antagonists, ${ }^{18)}$ and the thrombin inhibitor hirudin ${ }^{19}$ did not demonstrate positive effects after PTCA, use of the glycoprotein IIb / IIIa antagonist blocking the final common pathway of platelet aggregation resulted in fewer early ischemic complications $^{20)}$ and a lower frequency of clinical manifestations of restenosis. ${ }^{21)}$ However, no follow-up angiographic data are available.

The efficacy of cilostazol for prevention of restenosis after balloon angioplasty ${ }^{6,7,9)}$ and stent implantation ${ }^{8)}$ has been repeatedly demonstrated in controlled studies. Cilostazol (6-[4-(1-cyclohexyl-1H-tetrazol-5-yl)butoxy]-3,4-dihydro-2-(1H)-quinolinone) interferes with platelet function by increasing the cellular concentration of cyclic adenosine monophosphate (cAMP), this effect being mediated by inhibition of the cyclic nucleotide phosphodiesterase responsible for converting cAMP to 5'-adenosine monophosphate. ${ }^{22)}$ In a placebo controlled study in dogs, oral administration of cilostazol prevented thrombotic occlusion and intimal hyperplasia after placement of Gianturco Z-stents. ${ }^{23)}$ The oral dose applied had been predetermined to achieve 1-2 $\mu \mathrm{g}$ of plasma concentration, which is similar to the plasma concentration reached with $200 \mathrm{mg}$ per day of oral cilostazol in humans. ${ }^{24)}$

Ticlopidine (5-(2-chlorobenzyl)-4,5,6,7-tetrahydro (3,2-C) pyridine hydrochloride) acts as an antiplatelet agent, by activating platelet adenylate cyclase, enhancing the action of $\mathrm{PGE}_{1}$ to stimulate the cyclase, and blocking the inhibitory action of $\mathrm{PGE}_{2}$ on the cyclase. This results in elevation of cAMP in platelets, which again inhibits their aggregation. ${ }^{25,26)}$ Thus from the viewpoint of their end effects, ticlopidine and cilostazol have the same action. By avoiding platelet aggregation at the PTCA site, migration and proliferation of smooth muscle cells are suppressed and neointimal hyperplasia is obstructed.

The comparison of ticlopidine and cilostazol in the present study 
demonstrated no significant difference in restenosis rates. Unfortunately the result can not yet be extrapolated to general application, because the study population was relatively small and more patients are needed to obtain adequate statistical power. In addition, the time of initiation and duration of antiplatelet therapy must be considered. In the present study, it was started just after PTCA. Administration before the intervention may contribute to prevention of restenosis after PTCA, but this is clearly not an option with acute myocardial infarction patients. Actually, such individuals made up 6 of the ticlopidine group and 5 of the cilostazol group $(p=0.53)$. The optimal length of treatment remains to be defined.

Coronary artery stenting has achieved widespread acceptance in the field of coronary intervention. ${ }^{1,2)}$ Ochiai, et al. ${ }^{27)}$ reported a follow-up study after primary stenting for acute myocardial infarction. They demonstrated smaller late lumen loss and loss index in patients treated with cilostazol than those of ticlopidine, in line with our results, in which a tendency for better angiographic parameters in the cilostazol group was observed. Furthermore, a recent randomized trial of coronary artery stenting revealed cilostazol to have advantages over ticlopidine with respect to adverse drug reactions. ${ }^{28)}$ Warnings of serious adverse reactions such as thrombocytopenia, ${ }^{29)}$ leukopenia ${ }^{30)}$ and aplastic anemia ${ }^{31)}$ have been issued with use of ticlopidine. Although side effects in the ticlopidine group in the present study (elevated aminotransferase and skin rash) were mild, they required repeated monitoring of liver function and blood cell counts. In conclusion, the present prospective and randomized study demonstrated both ticlopidine and cilostazol to be effective for prevention of restenosis after PTCA. However cilostazol demonstrated less adverse drug reactions.

\section{REFERENCES}

1. Serruys PW, de Jaegere P, Kiemeneij F, et al. for the Benestent Study Group. A comparison of balloonexpandable-stent implantation with balloon angioplasty in patients with coronary artery disease. N Engl J Med 1994; 331: 489-95.

2. Fischman DL, Leon MB, Baim DS, et al. for the Stent Restenosis Study Investigators. A randomized comparison of coronary-stent placement and balloon angioplasty in the treatment of coronary artery disease. N Engl J Med 1994; 331: 496-501.

3. Ip JH, Fuster V, Israel D, Badimon L, Badimon J, Chesebro JH. The role of platelets, thrombin and hyperplasia in restenosis after coronary angioplasty. J Am Coll Cardiol 1991; 17: 77B-88B.

4. Schwartz RS, Holmes DR Jr, Topol EJ. The restenosis paradigm revisited: an alternative proposal for cellular mechanisms. J Am Coll Cardiol 1992; 20: 1284-93.

5. Yamaguchi T, Hamasaki S, Arima S, et al. Morphological effects on in-stent restenosis assessed by intravascular ultrasound imaging. Jpn Heart J 1999; 40: 109-18.

6. Take S, Matsutani M, Ueda H, et al. Effect of cilostazol in preventing restenosis after percutaneous transluminal coronary angioplasty. Am J Cardiol 1997; 79: 1097-9. 
7. Tsutsui M, Shimokawa H, Higuchi S, et al. Effect of cilostazol, a novel anti-platelet drug, on restenosis after percutaneous transluminal coronary angioplasty. Jpn Circ J 1996; 60: 207-15.

8. Yamasaki M, Hara K, Ikari Y, et al. Effects of cilostazol on late lumen loss after Palmaz-Schatz stent implantation. Cathet Cardiovasc Diagn 1998; 44: 387-91.

9. Tsuchikane E, Fukuhara A, Kobayashi T, et al. Impact of cilostazol on restenosis after percutaneous coronary balloon angioplasty. Circulation 1999; 100: 21-6.

10. Linderer T, Wunderlich W, Backs B, Noering J, Schroeder R. Edge detection in quantitative coronary arteriography (QCA): the impact of image zoom on edge positioning, accuracy, precision and discrimination. Comput Cardiol 1992; 99-102.

11. Califf RM, Fortin DF, Frid DJ, et al. Restenosis after coronary angioplasty: an overview. J Am Coll Cardiol 1991; 17: 2B-13B.

12. Haude M, Erbel R, Issa H, Meyer J. Quantitative analysis of elastic recoil after balloon angioplasty and after intracoronary implantation of balloon-expandable Palmaz-Schatz stents. J Am Coll Cardiol 1993; 21: 26-34.

13. Currier JW, Faxon DP. Restenosis after percutaneous transluminal coronary angioplasty: have we been aiming at the wrong target? J Am Coll Cardiol 1995; 25: 516-20.

14. Taylor RR, Gibbons FA, Cope GD, Cumpston GN, Mews GC, Luke P. Effects of low-dose aspirin on restenosis after coronary angioplasty. Am J Cardiol 1991; 68: 874-8.

15. Schwartz L, Bourassa MG, Lespérance J, et al. Aspirin and dipyridamole in the prevention of restenosis after percutaneous transluminal coronary angioplasty. N Engl J Med 1988; 318: 1714-9.

16. Urban P, Buller N, Fox K, Shapiro L, Bayliss J, Rickards A. Lack of effect of warfarin on the restenosis rate or on clinical outcome after balloon coronary angioplasty. Br Heart J 1988; 60: 485-8.

17. Ellis SG, Roubin GS, Wilentz J, Douglas JS Jr, King SB III. Effect of 18- to 24-hour heparin administration for prevention of restenosis after uncomplicated coronary angioplasty. Am Heart J 1989; 117: 777-82.

18. Serruys PW, Rutsch W, Heyndrickx GR, et al. For the Coronary Artery Restenosis Prevention on Repeated Thromboxane-Antagonism Study Group (CARPORT). Prevention of restenosis after percutaneous transluminal coronary angioplasty with thromboxane $\mathrm{A}_{2}$ - receptor blockade. A randomized, double-blind, placebo-controlled trial. Circulation 1991; 84: 1568-80.

19. Serruys PW, Herrman J-PR, Simon R, et al. For the Helvetica Investigators. A comparison of hirudin with heparin in the prevention of restenosis after coronary angioplasty. N Engl J Med 1995; 333: 757-63.

20. The EPIC Investigators. Use of a monoclonal antibody directed against the platelet glycoprotein IIb/IIIa receptor in high-risk coronary angioplasty. N Engl J Med 1994; 330: 956-61.

21. Topol EJ, Califf RM, Weisman HF, et al. on behalf of the EPIC investigators. Randomised trial of coronary intervention with antibody against platelet IIb/IIIa integrin for reduction of clinical restenosis: results at six months. Lancet 1994; 343: 881-6.

22. Umekawa H, Tanaka T, Kimura Y, Hidaka H. Purification of cyclic adenosine monophosphate phosphodiesterase from human platelets using new-inhibitor Sepharose chromatography. Biochem Pharmacol 1984; 33 : 3339-44.

23. Kubota Y, Kichikawa K, Uchida H, et al. Pharmacologic treatment of intimal hyperplasia after metallic stent placement in the peripheral arteries. An experimental study. Invest Radiol 1995; 30: 532-7.

24. Akiyama H, Kudo S, Shimizu T. The metabolism of a new antithrombotic and vasodilating agent, cilostazol, in rat, dog and man. Arzneim-Forsch / Drug Res 1985; 35(II): 1133-40.

25. Ashida S, Abiko Y. Inhibition of platelet aggregation by a new agent, ticlopidine. Thromb Haemost 1979; 40: 542-50.

26. Ashida S, Abiko Y. Mode of action of ticlopidine in inhibition of platelet aggregation in the rat. Thromb Haemost 1979; 41: 436-49.

27. Ochiai M, Eto K, Takeshita S, et al. Impact of cilostazol on clinical and angiographic outcome after primary stenting for acute myocardial infarction. Am J Cardiol 1999; 84: 1074-6.

28. Yoon Y, Shim W-H, Lee D-H, et al. Usefulness of cilostazol versus ticlopidine in coronary artery stenting. Am J Cardiol 1999; 84: 1375-80.

29. Bennett CL, Kiss JE, Weinberg PD, et al. Thrombotic thrombocytopenic purpura after stenting and ticlopidine. Lancet 1998; 352: 1036-7.

30. Gent M, Blakeley JA, Easton JD, et al. The Canadian American Ticlopidine Study (CATS) in thromboembolic stroke. Lancet 1989; 1: 1215-20. 
31. Ferrer F, De Arriba F, Moraleda JM, Vicente V. Aplastic anaemia induced by ticlopidine after placement of coronary artery stents. Therapeutic role of granulocyte colony-stimulating factor. Clin Drug Invest 1998; 15: 2612 . 
